\title{
Comparison of two CD40-ligand/interleukin-2 vaccines in patients with chronic lymphocytic leukemia
}

\author{
Fatma Visal Okur ${ }^{1}$, Eric Yvon ${ }^{1}$, Ettore Biagi ${ }^{1}$, Gianpietro Dotti ${ }^{1}$, George Carrum ${ }^{1}$, Helen \\ Heslop ${ }^{1}$, Martha P. Mims ${ }^{2}$, Joseph C. Fratantoni ${ }^{3,{ }^{,}}$, Madhusudan V. Peshwa ${ }^{3}$, Linhong $\mathrm{Li}^{3}$, \\ and Malcolm K. Brenner ${ }^{1}$ \\ ${ }^{1}$ Center for Cell and Gene Therapy, Baylor College of Medicine, The Methodist Hospital and \\ Texas Children 's Hospital, Houston, Texas, USA \\ ${ }^{2}$ Department of Medicine, Section of Hematology/Oncology, Baylor College of Medicine, Houston, \\ Texas, USA \\ ${ }^{3}$ MaxCyte Inc., Gaithersburg, Maryland, USA
}

\begin{abstract}
Background aims-Several studies have demonstrated that the immunogenicity of chronic lymphocytic leukemia (CLL) cells can be increased by manipulation of the CD40/CD40-ligand (CD40L) pathway. Although immunologic, and perhaps clinical, benefits have been obtained with an autologous CLL tumor vaccine obtained by transgenic expression of CD40L and interleukin (IL)-2, there is little information about the optimal gene transfer strategies.
\end{abstract}

Methods-We compared two different CLL vaccines prepared by adenoviral gene transfer and plasmid electroporation, analyzing their phenotype and immunostimulatory activity.

Results-We found that higher expression of transgenic CD40L was mediated by adenoviral gene transfer than by plasmid transduction, and that adenoviral transfer of CD40L was associated with up-regulation of the co-stimulatory molecules CD80 and CD86 and adhesion molecule CD54. In contrast, transgenic IL-2 secretion was greater following plasmid transduction. These phenotypic differences in the vaccines were associated with different functionality, both ex vivo and following administration to patients. Thus adenoviral vaccines induced greater activation of leukemia-reactive T cells ex vivo than plasmid vaccines. In treated patients, specific T-cell (T helper 1 (Th1) and T helper 2 (Th2) and humoral anti-leukemia responses were detected following administration of the adenoviral vaccine $(n=15)$, while recipients of the plasmid vaccine $(n=9)$ manifested only a low-level Th2 response. Progression-free survival at 2 years was $46.7 \%$ in the adenoviral vaccine recipients, versus $11.1 \%$ in those receiving plasmid vaccine

Conclusions-CLL vaccines expressing the same transgenes but produced by distinct methods of gene transfer may differ in the polarity of the immune response they induce in patients.

\section{Keywords}

CD40 ligand; chronic lymphocytic leukemia; electroporation; immunotherapy; tumor vaccine

\footnotetext{
(C) 2011 Informa Healthcare

Correspondence: Fatma Visal Okur, MD, Center for Cell and Gene Therapy, Baylor College of Medicine, 1102 Bates Street, Houston, TX 77030, USA., fatvis03@yahoo.com.

*Current address: Biologics Consulting Group Inc., 9412 Overlea Drive Rockville, MD 20850, USA.
} 


\section{Introduction}

Chronic lymphocytic leukemia (CLL) cannot be cured by current chemotherapy, and although allogeneic stem-cell transplantation may eradicate the disease, many patients are ineligible because of age. Even in suitable patients, there is a high treatment-related morbidity from infection and graft-versus-host disease (GvHD) (1-3). Most individuals with CLL have slowly progressive disease, and their malignant cells express differentiation antigens, surface idiotype immunoglobulins and major histocompatibility complex (MHC) class I and II antigens. In combination, these characteristics make the disease a good potential candidate for immunotherapy. Unfortunately CLL cells lack expression of many of the critical co-stimulatory molecules required for effective antigen presentation, and patients may also have numerical and functional defects in their T-cell compartment. These deficiencies impede the development of an effective anti-tumor immune response (4).

Genetic manipulation of the CD40/CD40-ligand (CD40L) pathway increases the immunogenicity of CLL cells by up-regulating co-stimulatory (CD80 and CD86) and adhesion (CD54) molecules and by inducing dendritic cell maturation (5-9). Previously, we and others have shown that expression of transgenic CD40L on autologous CLL cells can be produced by adenoviral gene transfer and will increase the immunogenicity of human CLL cells ex vivo and in vivo in patients with the disease $(7,10)$. We have also shown that the immunostimulatory properties of the $\mathrm{CD} 40 \mathrm{~L}$ vaccine are potentiated by co-transfecting a second adenoviral vector encoding interleukin (IL)-2 $(11,12)$. Initial clinical studies with this combination vaccine showed leukemia-specific T-cell and humoral immune responses and limited anti-tumor effects in vivo (13). Because of the complexities and expense of manufacture of viral vectors, and because of their lingering safety concerns, we determined whether it was feasible to substitute a physical means of gene transfer [using plasmid electroporation with the MaxCyte good manufacturing practice (GMP)-compliant device] for vaccine manufacture. We compared the properties of autologous CLL CD40L/IL-2 vaccines prepared by adenoviral gene transfer and by plasmid electroporation, analyzing their phenotype and immunostimulatory activity in individuals receiving one or other vaccine for treatment of CLL.

\section{Methods}

\section{Patient and treatment protocols}

This paper describes the in vitro and in vivo effects of two different CD40L/IL-2 CLL vaccines, one made using adenoviral vectors, the other using plasmid vectors, administered between 2003 and 2009. One or other vaccine was administered to a patient under concurrent phase I/II clinical protocols that were otherwise identical in inclusion and exclusion criteria and end-points, as described previously $(7,10,13)$. In brief, we assessed the toxicity (vaccine-related grade 3 or 4 toxicity, grade 2 allergic or autoimmune reactions; see http://ctep.info.nih.gov/ctc3/ctc.htm (accessed date: 14 December 2010) and disease response by standard criteria. Nine patients received between three and nine injections of the adenoviral vaccines between 2003 and 2005, as reported previously (13). Six additional patients received the same vaccine between 2007 and 2009 (total receiving adenoviral vaccine $=15$ ). Nine patients received six injections of the plasmid vaccines between 2004 and 2007. Both protocols were approved by the Institutional Review Board of the Baylor College of Medicine (Houston, TX, USA), the Food and Drug Administration, and the Recombinant DNA Advisory Committee of the National Institute of Health. An informed consent was obtained from each patient enrolled in the study according to the Helsinki Declaration. Patients were eligible for peripheral blood collection and vaccine preparation if they had a diagnosis of CLL with measurable disease of any stage, unless they were in Richter transformation. Eligibility to receive the vaccine required: (i) sufficient gene- 
modified CLL cells for six injections; (ii) a life expectancy of $\geq 10$ weeks; (iii) an absolute neutrophil count of $2500 / \mu \mathrm{L}$, an absolute lymphocyte count of $\geq 200 / \mu \mathrm{L}$, hemoglobin $\geq 8 \mathrm{~g} /$ $\mathrm{dL}$ and a platelet count of $250000 / \mu \mathrm{L}$; (iv) no treatment with other investigational agents within the last 4 weeks; (v) adequate liver (total bilirubin $\leq 1.5 \mathrm{mg} / \mathrm{dL}$, serum glutamic oxaloacetic transaminase (AST) (SGOT) $\leq 3$ times normal, normal prothrombin time) and renal (creatinine less than three times the normal for age or creatinine clearance $>80 \mathrm{~mL} /$ $\mathrm{min} / 1.73 \mathrm{~m}^{2}$ ) function; (vi) no immunosuppressive drugs within 6 weeks; and (vii) no autoimmune disorders. Individual patient characteristics are summarized in Table I.

\section{Adenoviral vectors and plasmids}

The adenoviral vectors encoding human IL-2 (hIL-2) and human CD40L (hCD40L) were constructed by recombining the pAVs6-hIL-2 and pAVs6-hCD40L plasmids with the ClaI fragment of Ad-dl327, an E3 deletion mutant of human adenovirus serotype 5 (Ad5) $(12,14,15)$. This vector lacks the E1a, E1b and E3 regions and thus is replication incompetent and devoid of genes to inhibit immune responses. Clinical-grade adenoviral vectors were expanded and tested according to local and federal requirements. The original vector titers were $6.67 \times 10^{11}$ infectious U/mL for Ad-hIL-2 and $7.75 \times 10^{10}$ infectious U/ $\mathrm{mL}$ for Ad-hCD40L. The hIL-2- and hCD40L-encoding plasmids (phIL-2 and phCD40L) were manufactured by Althea Technologies (San Diego, CA, USA) following current GMP (cGMP) guidelines.

\section{Vaccine preparation}

All manufacturing procedures followed current good tissue practice (cGTP) requirements. Mononuclear cells were isolated from peripheral blood by density-gradient centrifugation and stored at $-170^{\circ} \mathrm{C}$ in liquid nitrogen until further use. The percentage of leukemia cells was determined by immunostaining for CD5/CD19 and was always $>90 \%$.

\section{Adenoviral vaccines}

We have previously described the optimized protocol for preparing these vaccines (13). A certified human embryonic lung fibroblast cell line (MRC-5) (16) was obtained from the American Type Culture Collection (ATCC, Rockville, MD, USA). At the time of vaccine preparation, aliquots were thawed and cultured in RPMI-1640 (Hyclone, Perbio Science, Logan, UT, USA) supplemented with $10 \%$ heat-inactivated fetal calf serum (FCS; Hyclone) and $2 \mathrm{mmol} / \mathrm{L}_{\mathrm{L}}$-glutamine (BioWhit-taker, Walkersville, MD, USA) overnight. Next day they were trypsinized and separated into two aliquots. One aliquot was irradiated at $30 \mathrm{~Gy}$ and the remainder was transduced with the clinical-grade Ad-CD40L vector at a multiplicity of infection of 500 plaque-forming units (PFU)/cell and then irradiated at $30 \mathrm{~Gy}$. Both were cultured for 1-3 days until the MRC- 5 cells were $>50 \%$ confluent. CLL cells were then seeded onto the non-transduced MRC-5 cells or onto Ad-hCD40L-transduced MRC-5 cells and kept in culture for another $24 \mathrm{~h}$. CLL cells co-cultured with non-transduced MRC-5 cells were collected and transduced with the clinical-grade Ad-hIL-2 vector at a multiplicity of infection of 1000-2500 PFU/cell. An aliquot of these cells was maintained in culture for $72 \mathrm{~h}$ and IL-2 secretion was measured by enzyme-linked immunosorbent assay (ELISA). The remaining cells were frozen for further use (12). CLL cells co-cultured with hCD40Lexpressing MRC-5 cells were collected, and expression of CD40L, CD80, CD86 and CD54 (BD Bioscience, San Jose, CA, USA) checked by flow cytometry, before freezing the cells for further use (11).

\section{Plasmid vaccines}

We developed an electroporation protocol optimized for levels of CLL cell transduction/ transgene expression and for transduced cell viability (17-19). Frozen CLL cells were 
thawed and separated in two aliquots. They were resuspended at a cell concentration of 2-6 $\times 10^{8}$ cells $/ \mathrm{mL}$ in electroporation buffer (Hyclone). phIL-2 and phCD40L plasmid DNA solutions prepared at a final concentration of $0.44 \mathrm{mg} / \mathrm{mL}$ were then added to the cells. The cell/DNA mixtures were loaded into clinical-grade processing chambers and electroporated by the cGMP-compliant MaxCyte GT System (MaxCyte Inc., Gaithersburg, MD, USA). The processed CLL cells were incubated for $3-5 \mathrm{~h}$ at $37^{\circ} \mathrm{C}$ in $5 \% \mathrm{CO}_{2}$ before freezing for further use. Aliquots of hIL-2- and hCD40L-transfected CLL cells were removed to check IL-2 secretion by ELISA and CD40L expression by flow cytometry (17).

At the time of vaccination, aliquots of two vaccine components (CLL-hIL-2 and CLL$\mathrm{hCD} 40 \mathrm{~L}$ ) were thawed, mixed and irradiated at $30 \mathrm{~Gy}$, and resuspended in $0.5 \mathrm{~mL}$ of $1 \times$ phosphate-buffered solution (PBS) containing human albumin 5\%, and then injected subcutaneously into the upper arm of each patient, rotating arms between vaccinations.

\section{Viability of transduced cells}

We monitored the viability of each set of IL-2-secreting and CD40L-expressing CLL cell vaccines after thawing and irradiation (30 Gy), by taking an aliquot of $50 \mu \mathrm{L} 2 \times 10^{6} \mathrm{~B}-\mathrm{CLL}$ cells prior to injection and counting trypan blue-excluding cells over $72 \mathrm{~h}$.

\section{Activation of autologous $T$ cells from non-immunized patients by adenoviral- and plasmid- transduced vaccines}

Frozen CLL samples were thawed and separated into three aliquots. One aliquot was cocultured for $24 \mathrm{~h}$ with MRC- 5 cells transduced with adenovirus-expressing hCD40L, a second was co-cultured with MRC-5 cells transduced with adenovirus-expressing green flourescent protein (Ad5-GFP) to be used as a control, and the remaining cells were transfected with phCD40L plasmid via electroporation. We prepared autologous T cells isolated from each patient 's peripheral blood mononuclear cells (PBMC) before vaccination, and used positive selection with microbeads and magnetically activated cell sorting (MACS) technology to separate them into CD4 and CD8 subsets. These T cells were incubated for 5 days with $\gamma$-irradiated ( $40 \mathrm{~Gy}$ ) autologous CLL cells expressing transgenic CD40L of adenoviral or plasmid origin, and with CLL cells expressing GFP of adenoviral origin as a control, at a 1:1 ratio in 96-well plates. Their anti-leukemic reactivity was analyzed in a 36-h interferon (IFN)- $\gamma$ ELISPOT assay after restimulation with CD19selected unmanipulated autologous CLL cells. Cytokine production was measured in the 24$\mathrm{h}$ supernatants of these cultures using a cytometric bead assay (CBA) (BD Bioscience). T cells were also incubated for 5 days with the stimuli described above. They were then pulsed with $1 \mu \mathrm{Ci}\left[{ }^{3} \mathrm{H}\right]$ thymidine. Proliferation was measured $18 \mathrm{~h}$ later with a liquid scintillation analyzer (Packart, Meriden, CT, USA). CD80/86-blocking experiments were additionally done in ELISPOT and proliferation assays, to confirm that induction of leukemia-reactive $\mathrm{T}$ cells occurs through activation of the CD40/CD40L pathway. Pre-activated T cells were incubated with a mixture of unconjugated blocking CD80 and CD86 antibodies $(2 \mu \mathrm{g} / \mathrm{mL})$ (20) or with an isotype control antibody for $30 \mathrm{~min}$ at room temperature prior to co-culture for proliferation and ELISPOT assays.

\section{Treatment}

Patients in both the adenoviral and plasmid vaccine groups received subcutaneous injections of a fixed dose of irradiated (30 Gy) hIL-2 secreting autologous leukemic cells $\left(2 \times 10^{7}\right)$, with an escalating dose of irradiated ( $30 \mathrm{~Gy}) \mathrm{hCD} 40 \mathrm{~L}-$ expressing leukemic cells $\left(2 \times 10^{5}-2\right.$ $\times 10^{7}$ ). The first three doses of vaccine were administered at weekly intervals, followed by three to six additional injections at 2-week intervals if there was no evidence of disease progression (13). The patients were monitored for local and systemic toxicity using the National Cancer Institute Common Toxicity criteria (http://ctep.info.nih.gov/ctc3/ctc.htm 
(accessed date: 14 December 2010). Peripheral blood was collected every 1-2 weeks for the first 12 weeks, then monthly.

\section{Assessment of local and systemic immune responses after vaccine administration}

Nine of 15 patients treated with the adenoviral vaccine had injection-site biopsies at 1 week after the first and second injections, as did 9/9 patients receiving the plasmid vaccine. Immunohistochemical studies of formalin-fixed, paraffin-embedded sections used the DAKO envision system and autostainer (DAKO Cytomation, Glostrup, Denmark). Detailed analytic methods have been described elsewhere (13).

PBMC were phenotyped in all patients before and after each immunization and during follow-up examinations post-therapy by flow cytometry [fluorescence-activated cell sorter (FACS) Calibur cytometer; Becton Dickinson, San Jose, CA, USA] using antibodies to CD3, CD4, CD8, CD16, CD19, CD20, CD25, CD45RA, CD45RO, CD56, CD62L, CD69 and CCR7 (all purchased from BD Bioscience). Regulatory T cells in the peripheral blood of vaccinated CLL patients were evaluated by expression of cell-surface antigens CD4 and CD25 and intra cellular expression of FoxP3 by flow cytometry. The percentage of CD4 ${ }^{+}$ $\mathrm{CD} 25^{+}$FoxP $^{+}$regulatory $\mathrm{T}$ cells (Treg) in the $\mathrm{CD} 4^{+} \mathrm{T}$-lymphocyte population was determined by using a human FoxP3 staining set from eBioscience (San Diego, CA, USA).

Leukemia-specific humoral and cellular immune responses were evaluated before the first vaccination, then at 1-2 weeks after each injection. Autologous T cells were obtained from frozen PBMC collected before and after immunization, either using positive selection with CD4 and CD8 microbeads or negative selection with CD19 beads MACS Miltenyi Biotech, Bergish Gladbach Germany). The T cells were co-cultured with unmanipulated autologous CLL cells, collected, and frozen before immunization at a 1:5 T-cell:leukemic cell ratio in RPMI-1640 (BioWhittaker) supplemented with 5\% heat-inactivated human AB serum (Gemini BioProducts, Woodland, CA, USA) and 1\% L-glutamine (BioWhittaker). Control analyzes used each cell population alone and T cells co-cultured with allogeneic CLL cells. The MHC restriction of the leukemia-specific T-cell response was confirmed by incubating target cells with human HLA-A, HLA-B and HLA-C (clone W6/32), or HLA-DP, HLA-DQ and HLA-DR (clone CR3/43) antibodies (DAKO Cytomation), or isotype control antibody, all at $20 \mu \mathrm{L} / \mathrm{mL}$, for $30 \mathrm{~min}$ at room temperature (13); effector T cells were then added to the ELISPOT plates.

To detect leukemia-specific circulating IgM and $\operatorname{IgG}$ antibodies, autologous unmodified CLL cells collected before vaccination were incubated with diluted autologous plasma obtained before, during and after immunization. Plasma samples from healthy donors and unvaccinated CLL patients were used as controls. Flow cytometric analysis with a fluorescein isothiocyanate (FITC)-conjugated anti-human IgM (BD Bioscience) and phycoerythrin (PE)-conjugated anti-human IgG (BD-Bioscience) antibodies were used to detect antibody bound to human cells, as described previously $(13,21)$.

\section{Evaluation of clinical responses to vaccination}

Patients were evaluated pre-treatment, before each vaccination, and during post-treatment follow-up examinations every 4 weeks for 12 months, and then annually for 5 years, by physical examination with documentation of change in size of lymph nodes, spleen and liver, by differential blood count and serum chemistry.

\section{Statistical analysis}

The results of pre- and post-vaccination assays were compared by two-tailed paired $t$-test or Wilcoxon signed rank analyses, and those of adenoviral and plasmid vaccines by Pearson 
chi-square and Mann-Whitney $U$ analyzes using SPSS Statistics 18.0 software (SPSS Inc., Chicago, IL, USA). Time to progression was defined as the time from the initiation of vaccine therapy (first injection) to disease progression and/or initiation of chemotherapy. Survival probabilities were estimated by using the Kaplan-Meier method and tested using a $\log$ rank test. The results were presented as means and standard error of the mean (SE). $P$ values $<0.05$ were considered to be statistically significant.

\section{Results}

\section{Transgene expression by each vaccine}

Transduction with adenoviral vectors or transfection by plasmid electroporation led to expression of transgenes by the target tumor cells. Figure 1 shows that both adenoviral and plasmid transduction led to a significant increase in CD40L expression by CLL cells (from $0.5 \pm 0.2 \%$ to $79.8 \pm 4.3 \%, P=0.001$, for adenoviral, and from $1.3 \pm 0.85 \%$ to $55.5 \pm 5.1 \%$, $P=0.008$, for plasmid), although up-regulation was higher with the adenoviral vaccines $(P=$ $0.002)$. For the adenoviral vaccines, $\mathrm{CD} 40 \mathrm{~L}$ expression was accompanied by up-regulation of the co-stimulatory molecules CD80 $(P=0.001)$ and of CD86 $(P<0.001)$, probably as a consequence of activation of the CD40 pathway in the CLL cells by transgenic CD40L, rather than non-specific effects of adenoviral vector transduction (22). In plasmid vaccines, however, no significant up-regulation of CD80 and CD86 was detected, implying a reduced effect from transgenic CD40L. Adenoviral-induced CD40L expression and subsequent CD40 engagement on CLL cells also significantly increased their expression of the intercellular adhesion molecule, CD54 (ICAM-1), which facilitates effective antigen presentation (from $79.8 \pm 6.8 \%$ to $97.6 \pm 0.8 \%, P=0.007$ ). No such change was seen in plasmid-transduced vaccines (Figure 1a). Similarly, both routes of gene transfer led to IL-2 secretion by CLL cells, but, in contrast to CD40L expression, secretion was higher when CLL cells were transduced with the plasmid $\left(4806.6 \pm 1398.9 \mathrm{pg} / \mathrm{mL} / 24 \mathrm{~h} / 1 \times 10^{6}\right.$ cells) rather than with the adenoviral $\left(1199.6 \pm 82.6 \mathrm{pg} / \mathrm{mL} / 24 \mathrm{~h} / 1 \times 10^{6}\right.$ cells $)$ vector $(P=0.033)$.

Immediately after thawing for injection, viability of the B-CLL cells was higher in the CD40L adenoviral vaccines than in CD40L plasmid vaccines $(90 \pm 0.9 \%$ versus $56 \pm 8.7 \%$, $P=0.029)$, but the viability of the IL-2 vaccines was identical. Post-thawing, the viability of the plasmid-transduced cells declined significantly more rapidly in ex vivo culture (Figure 2).

\section{Activation of autologous $\mathrm{T}$ cells by adenoviral and plasmid vaccines}

To evaluate whether higher expression of CD40L and additional co-stimulatory molecules by adenoviral vaccines led to increased activation of leukemia-reactive T cells ex vivo, we co-cultured the patients' $\mathrm{T}$ cells with their own CD40L vaccines. Cytokine profiles were analyzed by measuring IL-2, IL-4, IL-5, IL-10, tumor necrosis factor (TNF)- $\alpha$ and IFN- $\gamma$ production in 24-h culture supernatants. Adenoviral vaccines induced greater increases in IL-2, IL-4, IL-5, TNF- $a$ and IFN- $\gamma$ release compared with plasmid vaccines (2.5-, 1.4-, 2-, 4.6- and 4.8-fold, respectively), while plasmid vaccines induced greater IL-10 release (1.8fold). Because of wide individual variation, only the differences between IFN- $\gamma$ concentrations reached overall significance $(P=0.048)$. We used proliferation and ELISPOT assays to assess further the ability of each vaccine to stimulate leukemia-reactive autologous T cells. CLL cells transduced with adeno-CD40L stimulated greater T-cell proliferation and more IFN- $\gamma$-producing T cells than CD40L plasmid-transduced CLL cells (Figure 3a,b). The absence of proliferation and IFN- $\gamma$ production when T cells were stimulated with CLL cells transduced with a GFP adenovirus excluded the possibility that the apparent differences between vaccines simply reflected a pre-existing memory T-cell response to adenoviral antigens. Blocking of CD80/86 molecules prior to co-culture of T cells with CLL 
cells led to inhibition of T-cell proliferation and IFN- $\gamma$ production. This finding supported the hypothesis that higher CD40L expression and subsequent up-regulation of costimulatory molecules (CD80 and CD86) by adenoviral vaccines induces a better leukemiareactive T-cell response.

\section{Toxicity}

Following administration of the vaccines, mild and self-limiting skin reactions, including local pain, erythema and swelling-associated myalgia and low-grade fever, were observed in four patients treated with adenoviral vaccines but in none of the patients receiving the plasmid vaccine. No patient developed signs of autoimmune disease or hepatic dysfunction.

\section{Local responses to vaccine administration}

We examined 18 injection-site biopsies from recipients of the adenoviral vaccine and 17 from recipients of the plasmid vaccine. These biopsy samples were obtained at 1 week after the first and second injections. A mild to moderate focal lymphocytic perivascular inflammatory reaction was observed in 14 out of 18 biopsies from the recipients of the adenoviral vaccine, and in eight out of 17 biopsies from recipients of plasmid vaccine (Pearson chi-square, $P=0.06$ ). As reported previously (13), the infiltrating immune cells were predominantly $\mathrm{CD} 4^{+} \mathrm{CD}^{+}$, and approximately $35 \%$ of the cells were $\mathrm{CD} 25^{+}$. No specimen contained more than rare CLL cells.

\section{Systemic responses to vaccine administration}

We compared the absolute numbers of circulating leucocytes, leukemic cells $\left(\mathrm{CD}^{+} \mathrm{CD} 19^{+}\right.$ $\mathrm{CD} 20^{+}$), T-lymphocyte subtypes and natural killer (NK) cells before vaccination and after the administration of sixth vaccine in each of the two treatment groups. In the recipients of the adenoviral vaccine, there were significant increases in absolute numbers of circulating non-leukemic lymphocytes, affecting both $\mathrm{CD}^{+} \mathrm{T}$ lymphocytes as a whole and the $\mathrm{CD} 8^{+}$, but not the $\mathrm{CD}^{+}$, subpopulation. NK cells were unchanged. In plasmid-vaccine recipients the absolute numbers of non-leukemic lymphocytes were unaltered (Figure 4). There was a small but statistically significant rise in the number of CLL cells in the circulation in the recipients of both vaccines.

As a component of our vaccine produced IL-2, and as more IL-2 was produced by the plasmid vaccine than the adenoviral vaccine, we also measured the numbers of regulatory $\mathrm{T}$ cells $\left(\mathrm{CD} 4^{+} \mathrm{CD} 25^{+} \mathrm{FoxP}^{+}\right)$before and after immunization in each group. The frequency of regulatory $\mathrm{T}$ cells in the recipients of either vaccine before the vaccine administration was similar (adenovirus $4.6 \pm 0.6 \%$ and plasmid $4.9 \pm 0.9 \%, P>0.05$ ) and there was no significant change with immunization in either group $(P=0.34)$ (Figure 5a,b).

\section{Leukemia-specific immune responses after vaccine administration}

We measured the induction of leukemia-specific T-cell responses by measuring IFN- $\gamma$ (as a marker of cytotoxic T lymphocytes (CTL) and Th1 cell recruitment) and IL-5 (as a marker of Th2 cell recruitment) release from T cells after co-culturing with autologous unmanipulated CLL cells. In the recipients of adenoviral vaccine, we detected significant increases in the frequencies of both IFN- $\gamma$ and IL-5 spot-forming T cells after the sixth injection (Figure 6a; IFN- $\gamma, P=0.004$, and IL-5, $P=0.038$ ). For the recipients of the plasmid vaccine, we noted a significant rise only in IL-5-producing spot-forming cells (Figure $6 \mathrm{~b} ; P=0.028$ ), to a level not significantly different from that observed in the adenoviral vaccine recipients. Hence the adenoviral vaccines induced a pattern of leukemia reactivity consistent with the presence of both a cytotoxic (Th1) and a helper (Th2) T-cell response, while the pattern of response induced by plasmid vaccines was consistent with a 
Th2 T-cell response. We confirmed that these antitumor responses were mediated by MHCrestricted T cells by showing inhibition with MHC class I- and II-blocking antibodies (Figure 6c).

We also measured changes in CLL-reactive $\operatorname{IgM}$ and $\operatorname{IgG}$ antibodies in vaccine recipients. An increase in leukemia-reactive $\operatorname{IgG}$ antibodies was detected in the plasma of three patients treated with adenoviral vaccine, but the levels were only 2- to 3-fold higher than pretreatment levels. No leukemia-specific antibody response was detected in the recipients of plasmid vaccine.

No reactivity was detected with plasma of three non-immunized patients or of three normal donors (data not shown). All leukemia-reactive cellular and humoral immune responses became undetectable $2-3$ months after the last vaccine.

\section{Clinical responses}

At the planned 10-week evaluation, all the plasmid and adenoviral vaccine recipients had stable disease. No substantive change in leukemia cell count was observed for any treated patient. The median time to progression was 9 months [95\% confidence interval (CI) $7.6-$ 10.4] for the plasmid group and 18 months (95\% CI 3.5 - 32.5) for the adenoviral group, with a 2-year progression-free survival (PFS) of $11.1 \%$ and $46.7 \%$, respectively $(P=0.024)$ (Table I). Although these differences appeared striking, this trial was not designed or powered to show the relative clinical efficacy of each vaccine.

\section{Discussion}

Broader application of tumor vaccines for the treatment of CLL will require measurable effectiveness, ease of manufacture and reduction in cost. For these reasons, electroporation of CLL cells with plasmids encoding immunostimulatory molecules such as CD40L and IL-2 has substantial potential advantages over adenoviral-mediated gene transfer. We have shown that a change in the method of gene transfer alters the levels of transgenes the vaccines express, and may substantially modify the immune responses they produce. In this particular study, plasmid transduction produced higher IL-2 but lower CD40L expression than an adenoviral vector, skewing the polarity of the immune response from Th1/Th2 to Th2.

Several pre-clinical and clinical studies have demonstrated that the immunogenicity of CLL cells can be increased by manipulation of the CD40/CD40L pathway $(7,9,12,13)$. CD40L expression is up-regulated after the initial activation of $\mathrm{T}$ cells produced by $\mathrm{T}$-cell receptor engagement of the MHC - peptide complex on the antigen-presenting cell (APC). CD40L activates APC, including B lymphocytes, through their CD40 receptor, which in turn upregulates their expression of the co-stimulatory molecules CD80 and CD86. These molecules stimulate the CD28 pathway in T cells, increasing IL-2 secretion and proliferation of antigen-specific $\mathrm{T}$ cells. Thus transgenic expression of CD40L by CLL cells will produce paracrine and perhaps autocrine stimulation of CD40 receptors on these cells, with subsequent up-regulation of co-stimulatory molecules CD80 and CD86. The effect should be to enhance their antigen-presenting capacity and generate leukemia-specific $\mathrm{T}$ cells by breaking the anergic state of circulating T cells in CLL $(6,23)$. These observations have led to clinical trials evaluating the feasibility of using CD40L-expressing autologous CLL cells as a tumor vaccine, which have produced clinical responses, including a transient but consistent decrease of circulating leukemic cell counts and reduction in lymphadenopathy (10). We have reported previously a modification of this approach, combining transgenic human CD40L-expressing CLL cells with transgenic IL-2-secreting CLL cells, in an attempt to expand by IL-2 stimulation the tumor-reactive T cells generated in response to the 
CD40L-transgenic CLL cells. This approach elicited a superior leukemia-reactive T-cell response compared with either molecule alone (12), and subcutaneous injections of CLL cells expressing these molecules induced transient but specific T-cell and humoral antileukemia responses in seven of nine patients, with transient clinical responses detected in three patients (13) and one complete response (24).

The above studies all used adenovirus as the transfer vector. Adenoviruses have several advantages over alternative vectors. They transduce even resting cells to produce generally high levels of transgene expression, and, because they are non- integrating, they have low potential for genotoxicity. Although adenovector-mediated transgene expression may be short lived and the antigens of the adenoviral vector may themselves induce an immune response to the transduced cells, neither of these limitations is problematic for a tumor vaccine. Adenoviruses, however, do not readily transduce CLL cells, which lack the coxsackie/adenovirus receptor (CAR). Thus high, and somewhat toxic, numbers of adenoviral particles are required $(11,25)$. Although this limitation can be overcome, for example by modifying the serotype of the adenoviral vector or using 'feeder cells' $(11,25)$, all these manipulations add to complexity and cost. Finally, manufacture and testing of clinical-grade adenoviral vectors is inherently complex, slow and expensive, and clearly impedes broader exploration and implementation of the approach.

In contrast, producing vaccine by physical transduction of cDNA plasmids using electroporation is relatively low cost, requires much less time and does not require specific cell-surface receptors for efficient gene delivery. The availability of validated clinical-grade transfection devices allows a rapid, rigorously controlled and constantly monitored transfection process $(17,26)$. Non-dividing cells can be transduced and, as integration is rare, genotoxicity is low. By adjusting the concentration of plasmid and the characteristics of the electroporation itself, generally high levels of transgene expression can be obtained, although, as we show here, these are not always equivalent to the values obtained using adenoviral vectors.

Although both hCD40L and hIL-2 were expressed by CLL cells irrespective of the method of transduction, the levels of $\mathrm{CD} 40 \mathrm{~L}$ were higher for the adenoviral vaccines, while IL-2 was higher in the electroporated cells. Simply increasing plasmid concentrations or the multiplicity of infection of the adenoviral vector did not modify this pattern, leading instead to greater toxicity in the transduced population. Although we do not know the mechanism of these differences, the lower level of $\mathrm{CD} 40 \mathrm{~L}$ expression obtained using the plasmid vector was associated with both phenotypic and functional consequences in vitro. The plasmidderived tumor vaccines had lower expression of CD80 and CD86, the ligands for the T-cell co-stimulatory molecule CD28. We suggest that this difference is because of reduced autocrine/paracrine CD40 activation by the lower level of CD40L expressed on plasmidtransduced CLL cells and the shorter time - course of vaccine manufacture ( $5 \mathrm{~h}$ versus 3 days). The importance of CD80 and CD86 in enhancing immunostimulation by the adenoviral vaccine is shown by the inhibitory effects of CD80/86 monoclonal antibodies on T-cell proliferation and IFN- $\gamma$ secretion following exposure to the Ad-CD40L-transduced CLL cells. As a consequence of these phenotypic differences, co-culture of CD40L plasmidtransduced CLL cells with $\mathrm{T}$ lymphocytes led to reduced T-cell proliferation and cytokine release compared with co-culture with CD40L adenovirus CD40L-transduced CLL cells (Ad-CLL).

While these ex vivo data suggested that the CD40L adenoviral vaccine might have superior $\mathrm{T}$-cell activating properties, it could be postulated that in vivo the higher local concentrations of IL-2 produced by the plasmid-transduced CLL cells would compensate for a lower baseline level of CD40L-mediated stimulation of T-cell proliferation. As this 
potential compensatory benefit cannot be modeled accurately in vitro, we proceeded with phase I/II trials of each type of vaccine in separate, although comparable, patient cohorts (Table I).

Our subsequent in vivo observations of immunized patients indicated that differences in IL-2 and CD40L expression in the vaccines administered were reflected in differences both in the intensity of the immune response and its polarity. Based on patterns of cytokine production, the adenoviral vaccine induced both a Th1 and Th2 immune response, while the plasmid vaccine generated primarily a Th2 anti-tumor response. This trial was not designed or powered to show the relative clinical efficacy of each vaccine. We observed, however, that while the number of circulating B-CLL cells after six vaccinations was higher than the pre-treatment level in groups receiving either adenoviral or plasmid vaccines (Figure 4), the time to progression and 2-year progression-free survival for the adenoviral vaccine group was nonetheless longer than for the recipients of the plasmid vaccine, so that early analysis of disease response may not predict ultimate outcome.

There may be several explanations for the differences we observed in the polarity and intensity of the in vivo immune response, which are not mutually exclusive. One is that the plasmid vaccine survived more briefly in vivo than the adenoviral vaccine, so that the duration of stimulation from CD40L and IL-2 was shorter. Certainly the CD40L plasmid vaccine survived less well after thawing when cultured ex vivo than the adenoviral vaccine. This attenuated viability of electroporated CLL cells is probably a result of continuing apoptosis induced by the uptake of double-stranded DNA, as Li et al. (17) observed that 60 $-70 \%$ of DNA-electroporated CLL cells were apoptotic, while the viability of FITC dextran electroporated CLL was no different from unmanipulated controls. Unfortunately, we have no means of determining whether a similar difference in the survival of the two vaccines occurs in vivo; our injection-site biopsies were all at day 7 after vaccination, by which time visible tumor vaccine cells had disappeared, irrespective of whether the product was adenovirus- or plasmid-transduced CLL cells. Even if the plasmid-transduced tumor cells survive as well in vivo as the adenoviral vaccines, their lower expression of CD40L and their $\mathrm{CD} 80 / 86^{-}$phenotype may diminish their capacity to stimulate $\mathrm{CD} 8 / \mathrm{Th} 1$ cells, favoring instead expansion of the Th2 subset (27).

An alternative explanation for the difference in the immune responses generated by plasmid and adenoviral vaccine is that, independent of the reduced expression of CD40L, the greater production of IL-2 by the plasmid vaccines alters the intensity and polarity of the immune response as a result of the increased recruitment of CD25 ${ }^{+}$Treg cells. While such an explanation may be feasible, we could not find supporting evidence, as flow cytometric analysis of peripheral blood from recipients of adenoviral or plasmid vaccines showed no differences in $\mathrm{CD} 4{ }^{+} \mathrm{CD} 25^{+} \mathrm{FoxP} 3{ }^{+}$cells before, during or after immunization. We cannot, however, exclude differences in the functional activity of Treg cells as a result of the limited availability of Treg cells from these clinical samples.

In conclusion, CLL vaccines produced by electroporation with plasmid are preferable to vaccines produced by adenoviral vectors in terms of simplicity, cost and speed, but it cannot be assumed that there will be functional equivalence between the cellular products, even when both express the same trans-genes. Fortunately, our data show that ex vivo measures of phenotype and function appear to predict subsequent in vivo activity, and we propose that this predictive power should ultimately allow optimization of the logistically simpler plasmid-derived tumor vaccines and render there in vivo potency equivalent to vaccines obtained by adenoviral-mediated gene transfer (13). 


\section{Acknowledgments}

The work described in this manuscript was supported by NIH-NCI SPORE P50 CA 126752.

\section{References}

1. Chiorazzi N, Rai KR, Ferrarini M. Chronic lymphocytic leukemia. N Engl J Med. 2005; 352:80415. [PubMed: 15728813]

2. Jabbour E, Keating MJ, Champlin RE, Khouri IF. Stem cell transplantation for chronic lymphocytic leukemia: should not more patients get a transplant? Bone Marrow Transplant. 2004; 34:289-97. [PubMed: 15220957]

3. Khouri IF, Keating MJ, Vriesendorp HM, Reading CL, Przepiorka D, Huh YO, et al. Autologous and allogeneic bone marrow transplantation for chronic lymphocytic leukemia: preliminary results. J Clin Oncol. 1994; 12:748-58. [PubMed: 8151318]

4. Mellstedt H, Choudhury A. T and B cells in B-chronic lymphocytic leukaemia: Faust, Mephistopheles and the pact with the Devil. Cancer Immunol Immunother. 2006; 55:210-20. [PubMed: 15906026]

5. Buhmann R, Nolte A, Westhaus D, Emmerich B, Hallek M. CD40-activated B-cell chronic lymphocytic leukemia cells for tumor immunotherapy: stimulation of allogeneic versus autologous T cells generates different types of effector cells. Blood. 1999; 93:1992-2002. [PubMed: 10068672]

6. Dilloo D, Brown M, Roskrow M, Zhong W, Holladay M, Holden W, et al. CD40 ligand induces an antileukemia immune response in vivo. Blood. 1997; 90:1927-33. [PubMed: 9292526]

7. Kato K, Cantwell MJ, Sharma S, Kipps TJ. Gene transfer of CD40-ligand induces autologous immune recognition of chronic lymphocytic leukemia B cells. J Clin Invest. 1998; 101:1133-41. [PubMed: 9486984]

8. McLellan AD, Sorg RV, Williams LA, Hart DN. Human dendritic cells activate T lymphocytes via a CD40:CD40 ligand-dependent pathway. Eur J Immunol. 1996; 26:1204-10. [PubMed: 8647193]

9. Messmer D, Kipps TJ. CD154 gene therapy for human B-cell malignancies. Ann NY Acad Sci. 2005; 1062:51-60. [PubMed: 16461788]

10. Wierda WG, Cantwell MJ, Woods SJ, Rassenti LZ, Prussak CE, Kipps TJ. CD40-ligand (CD154) gene therapy for chronic lymphocytic leukemia. Blood. 2000; 96:2917-24. [PubMed: 11049967]

11. Biagi E, Yvon E, Dotti G, Amrolia PJ, Takahashi S, Popat U, et al. Bystander transfer of functional human CD40 ligand from gene-modified fisbroblasts to B-chronic lymphocytic leukemia cells. Hum Gene Ther. 2003; 14:545-59. [PubMed: 12718765]

12. Takahashi S, Rousseau RF, Yotnda P, Mei Z, Dotti G, Rill D, et al. Autologous antileukemic immune response induced by chronic lymphocytic leukemia B cells expressing the CD40 ligand and interleukin 2 transgenes. Hum Gene Ther. 2001; 12:659-70. [PubMed: 11426465]

13. Biagi E, Rousseau R, Yvon E, Schwartz M, Dotti G, Foster A, et al. Responses to human CD40 ligand/human inter-leukin-2 autologous cell vaccine in patients with B-cell chronic lymphocytic leukemia. Clin Cancer Res. 2005; 11:6916-23. [PubMed: 16203783]

14. Bowman L, Grossmann M, Rill D, Brown M, Zhong WY, Alexander B, et al. IL-2 adenovectortransduced autologous tumor cells induce antitumor immune responses in patients with neuroblastoma. Blood. 1998; 92:1941-9. [PubMed: 9731051]

15. Gauchat JF, Aubry JP, Mazzei G, Life P, Jomotte T, Elson G, et al. Human CD40-ligand: molecular cloning, cellular distribution and regulation of expression by factors controlling $\operatorname{IgE}$ production. FEBS Lett. 1993; 315:259-66. [PubMed: 7678552]

16. Jacobs JP, Jones CM, Baille JP. Characteristics of a human diploid cell designated MRC-5. Nature. 1970; 227:168-70. [PubMed: 4316953]

17. Li LH, Biagi E, Allen C, Shivakumar R, Weiss JM, Feller S, et al. Rapid and efficient nonviral gene delivery of CD154 to primary chronic lymphocytic leukemia cells. Cancer Gene Ther. 2006; 13:215-24. [PubMed: 16082377]

18. Li LH, Shivakumar R, Feller S, Allen C, Weiss JM, Dzekunov S, et al. Highly efficient, large volume flow electroporation. Technol Cancer Res Treat. 2002; 1:341-50. [PubMed: 12625759] 
19. Fratantoni JC, Li L, Liu LN, Peshwa MV, Brenner M, Yvon E, et al. A practical approach for achieving clinical immunotherapy of CLL with hCD40L- and hIL-2- expressing autologous tumor cells (ASH Annual meeting abstracts). Blood. 2005; 106:450.

20. Balbo P, Silvestri M, Rossi GA, Crimi E, Burastero SE. Differential role of CD80 and CD86 on alveolar macrophages in the presentation of allergen to T lymphocytes in asthma. Clin Exp Allergy. 2001; 31:625-36. [PubMed: 11359432]

21. Rousseau RF, Haight AE, Hirschmann-Jax C, Yvon ES, Rill DR, Mei Z, et al. Local and systemic effects of an allogeneic tumor cell vaccine combining transgenic human lymphotactin with interleukin-2 in patients with advanced or refractory neuroblastoma. Blood. 2003; 101:1718-26. [PubMed: 12406881]

22. Ranheim EA, Kipps TJ. Activated T cells induce expression of B7/BB1 on normal or leukemic B cells through a CD40-dependent signal. J Exp Med. 1993; 177:925-35. [PubMed: 7681471]

23. Cardoso AA, Seamon MJ, Afonso HM, Ghia P, Boussiotis VA, Freeman GJ, et al. Ex vivo generation of human anti-pre-B leukemia-specific autologous cytolytic T cells. Blood. 1997; 90:549-61. [PubMed: 9226154]

24. Foster AE, Okur FV, Biagi E, Lu A, Dotti G, Yvon E, et al. Selective depletion of a minor subpopulation of B-chronic lymphocytic leukemia cells is followed by a delayed but progressive loss of bulk tumor cells and disease regression. Mol Cancer. 2009; 8:106. [PubMed: 19922650]

25. Cantwell MJ, Sharma S, Friedmann T, Kipps TJ. Adenovirus vector infection of chronic lymphocytic leukemia B cells. Blood. 1996; 88:4676-83. [PubMed: 8977261]

26. Fratantoni JC, Dzekunov S, Singh V, Liu LN. A non-viral gene delivery system designed for clinical use. Cytotherapy. 2003; 5:208-10. [PubMed: 12850788]

27. Mackey MF, Barth RJ Jr, Noelle RJ. The role of CD40/CD154 interactions in the priming, differentiation, and effector function of helper and cytotoxic T cells. J Leukoc Biol. 1998; 63:41828. [PubMed: 9544571] 

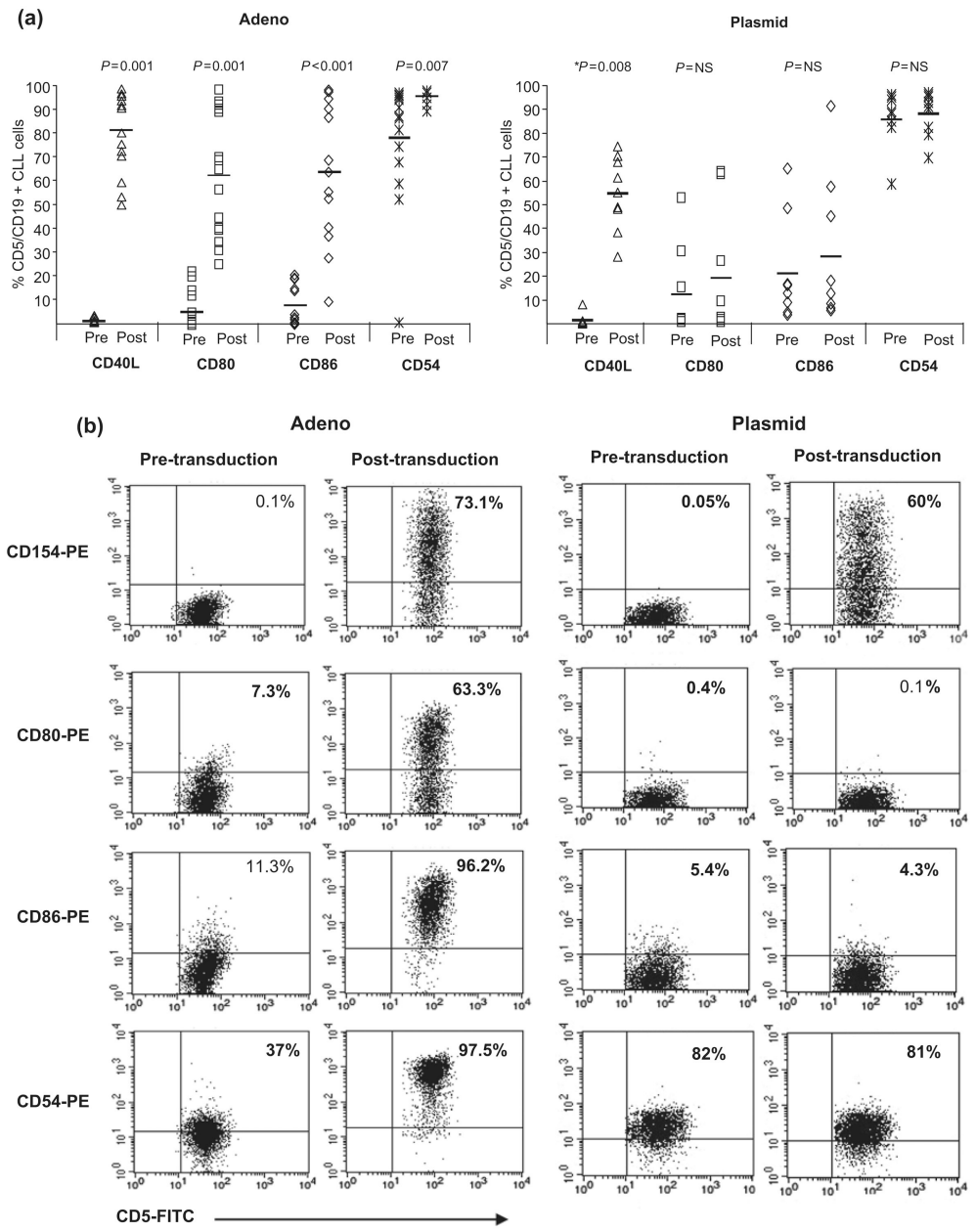

Figure 1.

(a) The percentage of viable CLL cells gated on CD5/CD19, expressing CD40L, CD80, CD86 and CD54 before (pre-) and after (post-) manipulation for preparation of CD40L components of adenovirus and plasmid vaccines. (b) Detail of B-CLL cell expression of CD54, CD80, CD86 and CD40L before and after transduction with adenoviral or plasmid vectors (a representative sample). $* P<0.05$ 


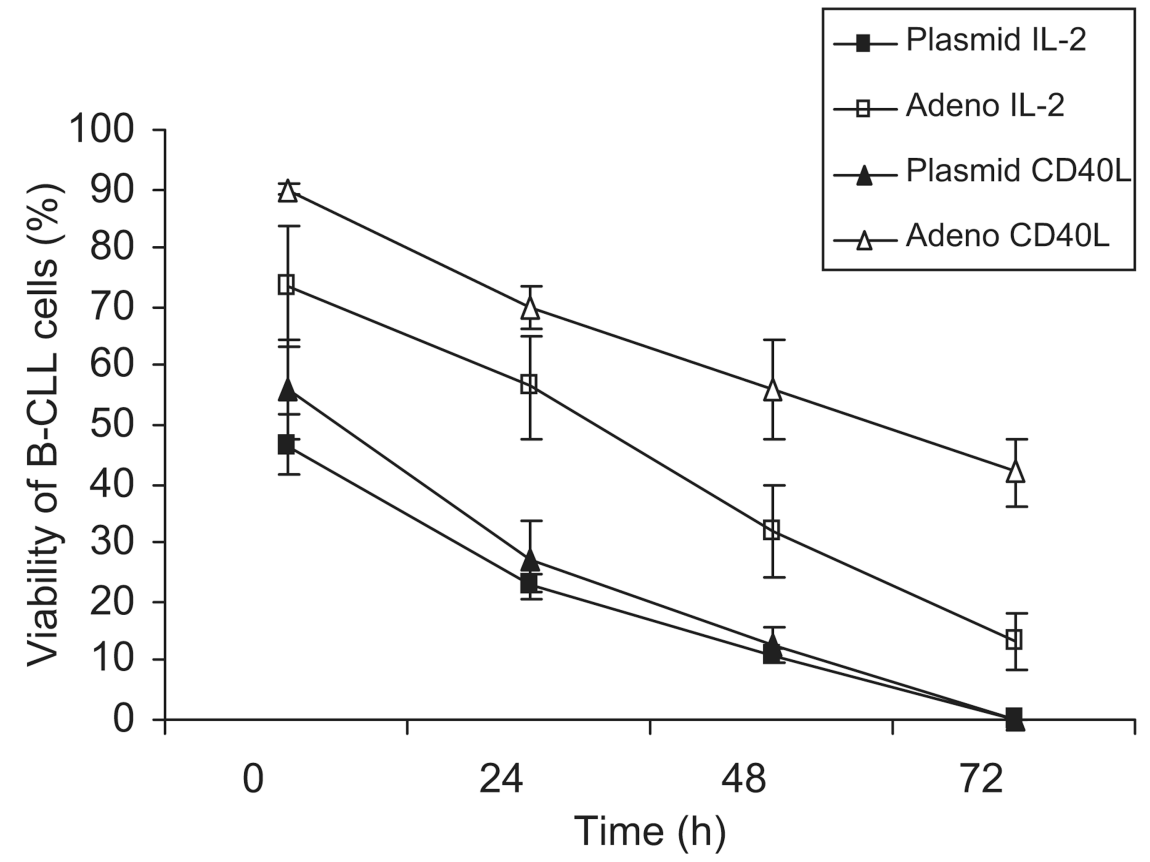

Figure 2.

Viability of IL-2-secreting and CD40L-expressing CLL cells after thawing and irradiation (30 Gy) $(n=4)$. CLL cells were monitored for viability using trypan blue count on aliquots of $50 \mu \mathrm{L}$ of $2 \times 10^{6} \mathrm{CLL} / \mathrm{mL}$ using an inverted microscope. 
(a)

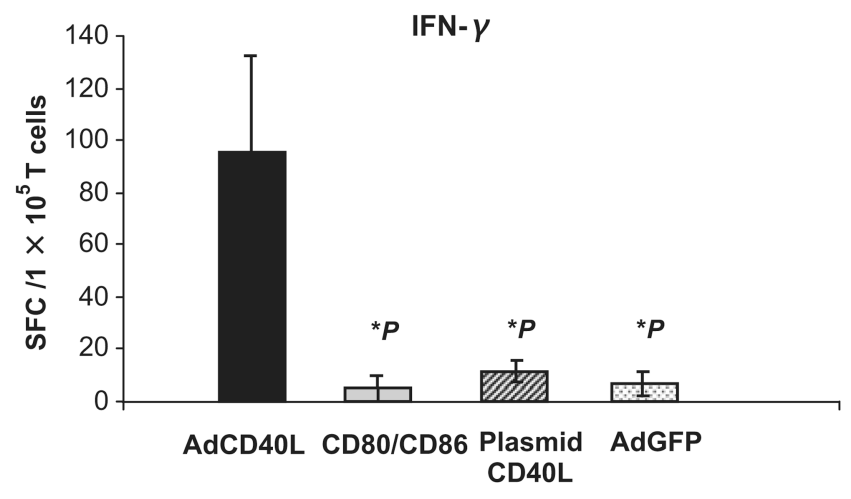

(b)

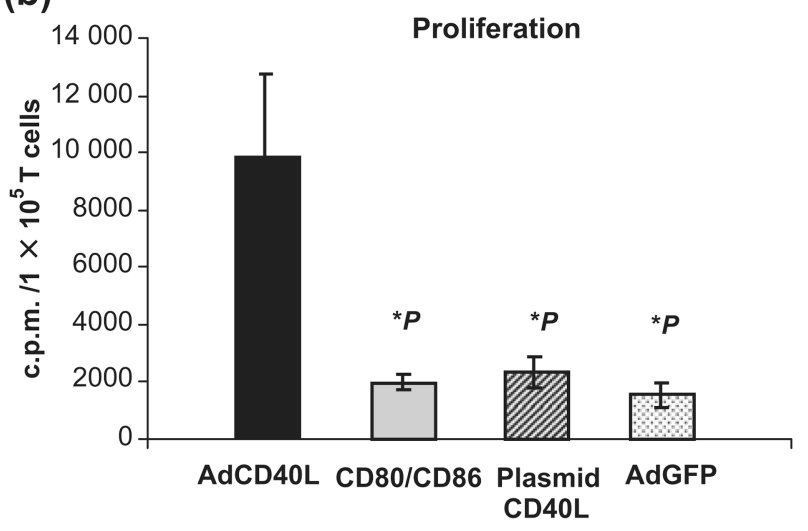

Figure 3.

(a) CD40L-expressing CLL cells induce activation and proliferation of leukemia-reactive CD4/CD8T lymphocytes. T lymphocytes obtained before treatment were co-cultured with 40-Gy irradiated CD40L expressing autologous CLL cells (adenovirus and plasmid) and CLL cells expressing Ad-GFP as a control for 5 days. Antileukemic reactivity of these T cells was analyzed with a 36-h ELISPOT assay $(n=5)$. (b) The proliferation of leukemiareactive $\mathrm{T}$ lymphocytes was tested in a mixed lymphocyte reaction with autologous PBMC. T cells were incubated at a 1:1 ratio with 40-Gy irradiated stimulator cells (CLL cells). Proliferation was measured on day 5 by $\left[{ }^{3} \mathrm{H}\right]$ thymidine incorporation $(n=6)$. CD80 and CD86 blocking significantly decreased anti-leukemic reactivity and cell proliferation. SFC: spot-forming cells; c.p.m.: cyles per minute. $* P<0.05$. 


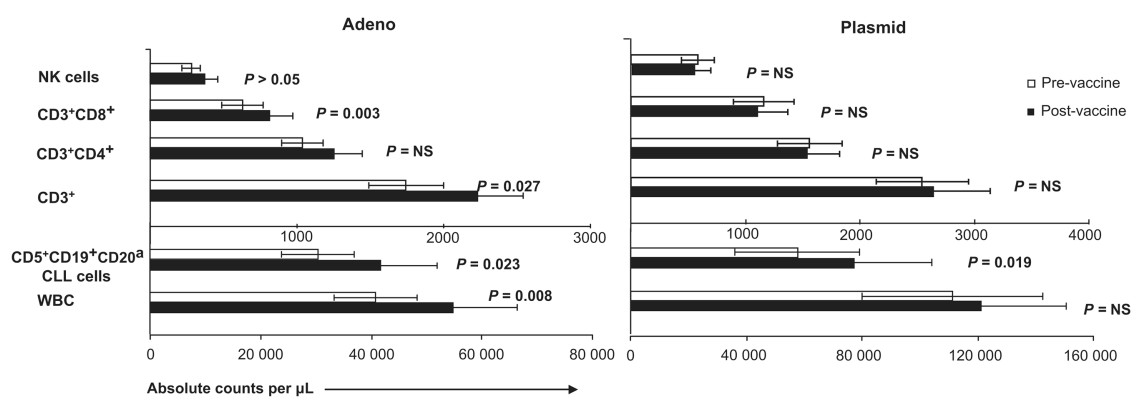

Figure 4.

Flow cytometric analysis of leucocyte and lymphocyte subpopulations before (open squares) and after (closed squares) immunization (sixth injection). Absolute circulating leucocyte, leukemic cell $\left(\mathrm{CD}^{+} \mathrm{CD} 19^{+} \mathrm{CD} 20^{+}\right)$and lymphocyte counts showing helper $\left(\mathrm{CD}^{+} \mathrm{CD}^{+}\right)$, cytotoxic $\left(\mathrm{CD}^{+} \mathrm{CD}^{+}\right)$and $\mathrm{NK}$ cell $\left(\mathrm{CD} 16^{+} \mathrm{CD}^{+} 6^{+}\right)$subpopulations in adenoviral vaccine recipients and plasmid vaccine recipients. WBC: white blood cells. 
(a)

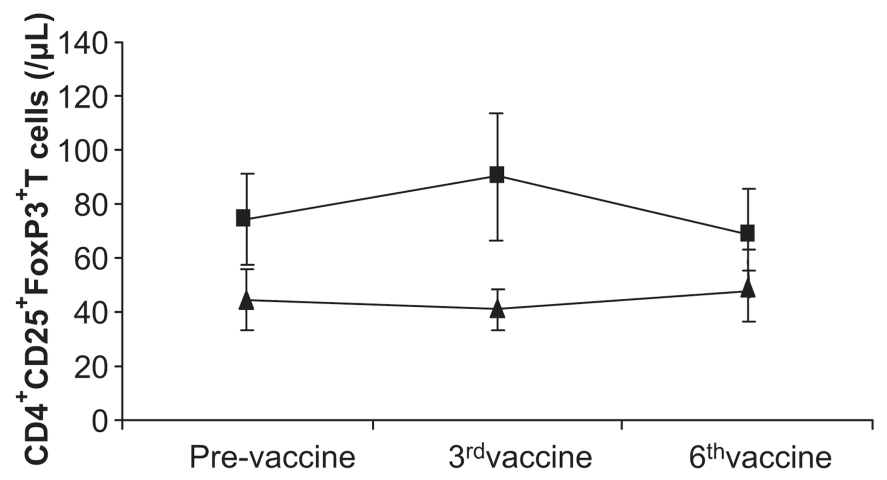

(b)

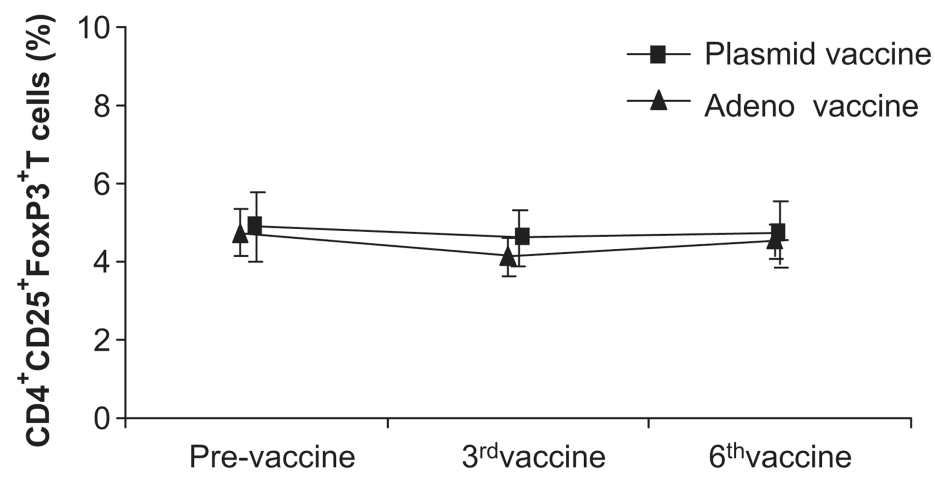

Figure 5.

Numbers and percentages of regulatory $\mathrm{T}$ cells present in the peripheral blood of treated patients remained unchanged before and after vaccination. (a) Absolute number and (b) mean percentage of $\mathrm{CD}^{+}{ }^{+} \mathrm{CD} 25^{+} \mathrm{FoxP}^{+}$(gated on CD4) regulatory $\mathrm{T}$ cells detected in the peripheral blood pre-vaccine, and 2 weeks after the third and sixth vaccine administrations. 

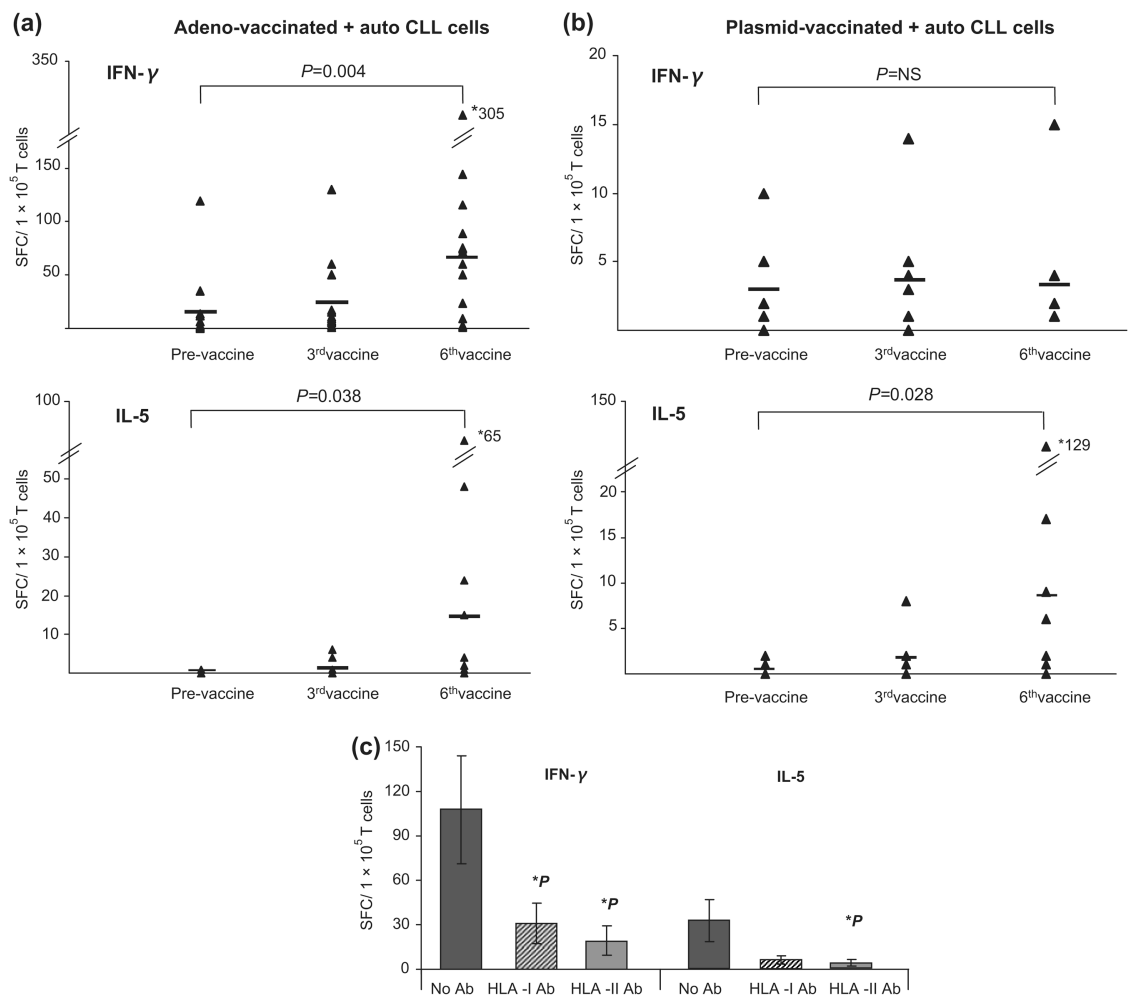

Figure 6.

ELISPOT results showing IFN- $\gamma$ (cytotoxic T-cell and Th1-cell profiles) and IL-5 (Th2 profile) release from activated $\mathrm{T}$ lymphocytes obtained before and after injections in (a) adenoviral vaccine, (b) plasmid vaccine recipients and (c) before and after human leucocyte antigen (HLA) class I (HLA-A, B and C) blocking antibody and HLA class II (HLA-DP, DQ and -DR) antibodies blocking in adenoviral vaccine recipients. Mean numbers of IFN- $\gamma$ and IL-5 spot-forming cells (SFC) among $1 \times 10^{5} \mathrm{~T}$ cells isolated from peripheral blood before and 1-2 weeks after the third and sixth vaccine administrations. T cells were cocultured with unmanipulated autologous leukemia cells at a 1:5 effector:target ratio for $36 \mathrm{~h}$. $* P<0.05$. 


\section{Table I}

Characteristics of patients and clinical outcome.

\begin{tabular}{|c|c|c|c|c|}
\hline Patient/protocol & Age (years)/gender & Stage (Rai) & Previous therapy & $\begin{array}{c}\text { Time to progression/chemotherapy } \\
\text { (months) }\end{array}$ \\
\hline \multicolumn{5}{|l|}{ Adenoviral } \\
\hline P1126 & $46 / M$ & 3 & Yes & 28 \\
\hline P1256 & $49 / \mathrm{M}$ & 4 & No & 7 \\
\hline $\mathrm{P} 1300$ & $65 / \mathrm{M}$ & 2 & No & Stable at 57 \\
\hline $\mathrm{P} 1232$ & $51 / \mathrm{F}$ & 3 & Yes & 16 \\
\hline P1336 & $54 / \mathrm{M}$ & 4 & No & 18 \\
\hline $\mathrm{P} 1253$ & $71 / \mathrm{M}$ & 1 & No & 8 \\
\hline P1393 & $67 / \mathrm{M}$ & 2 & No & 6 \\
\hline P1335 & $63 / F$ & 2 & No & 8 \\
\hline P1233 & $53 / \mathrm{M}$ & 2 & Yes & 43 \\
\hline P1991 & $39 / \mathrm{M}$ & 4 & No & 8 \\
\hline P1964 & $49 / \mathrm{M}$ & 4 & No & Stable at 37 \\
\hline P2090 & $71 / \mathrm{M}$ & 4 & No & 27 \\
\hline $\mathrm{P} 2220$ & $52 / \mathrm{M}$ & 2 & No & Stable at 24 \\
\hline $\mathrm{P} 2250$ & $70 / \mathrm{M}$ & 1 & No & Stable at 24 \\
\hline P2347 & $50 / \mathrm{F}$ & 2 & No & 13 \\
\hline \multicolumn{5}{|l|}{ Plasmid } \\
\hline P1376 & $69 / \mathrm{M}$ & 2 & No & 7 \\
\hline P1477 & $82 / \mathrm{M}$ & 1 & No & 10 \\
\hline P1529 & $61 / \mathrm{M}$ & 1 & No & 8 \\
\hline P1336 & $54 / \mathrm{M}$ & 1 & Yes & 9 \\
\hline P1126 & $47 / \mathrm{M}$ & 3 & Yes & 9 \\
\hline P1232 & $52 / \mathrm{F}$ & 1 & Yes & 5 \\
\hline P1593 & $69 / \mathrm{M}$ & 4 & No & 6 \\
\hline P1954 & $73 / \mathrm{M}$ & 0 & No & 24 \\
\hline P2085 & $47 / \mathrm{M}$ & 2 & No & Stable at 9 \\
\hline $\begin{array}{l}\text { P-value for difference between vaccine } \\
\text { groups }\end{array}$ & NS & NS & NS & 0.024 \\
\hline
\end{tabular}

M, male; F, female; NS, non-significant. $P$-values $<0.05$ considered statistically significant; Rai: staging system for CLL. 